The Astrophysical Journal, 496:226-234, 1998 March 20

(C) 1998. The American Astronomical Society. All rights reserved. Printed in U.S.A.

\title{
THE EFFECT OF STAR FORMATION ON MOLECULAR CLOUDS IN DWARF IRREGULAR GALAXIES: IC 10 AND NGC 6822
}

\author{
Glen R. Petitpas and Christine D. Wilson \\ Department of Physics and Astronomy, McMaster University, 1280 Main Street West, Hamilton, Ontario, Canada L8S 4M1; \\ petipa@physics.mcmaster.ca, wilson@physics.mcmaster.ca \\ Received 1997 March 26; accepted 1997 October 24
}

\begin{abstract}
We have observed the ${ }^{12} \mathrm{CO} J=2-1$ and $J=3-2$ lines at a few locations in the dwarf irregular galaxies IC 10 and NGC 6822 using the James Clerk Maxwell Telescope. In addition, we have observed the ${ }^{13} \mathrm{CO} J=2-1$ line for IC 10 and the first detection of the ${ }^{13} \mathrm{CO} J=3-2$ transition in a Local Group galaxy. The CO line ratios in IC 10 are uniform and are consistent with the average line ratios observed in M33 at the $1 \sigma$ level. These low-metallicity environments appear to be porous to UV radiation and allow for more efficient heating of molecular gas by nearby $\mathrm{H}$ II regions. The ${ }^{12} \mathrm{CO} J=3-2 / J=2-1$ ratio for the molecular cloud in NGC 6822 is higher than those found for IC 10 and M33 and suggests that the ${ }^{12} \mathrm{CO}$ emission is optically thin in this region. This high line ratio is likely the result of its location inside a large $\mathrm{H}$ II region with low metallicity and low gas content, and requires a hydrogen density greater than $10^{4} \mathrm{~cm}^{-3}$ and a kinetic temperature greater than $100 \mathrm{~K}$. The ${ }^{12} \mathrm{CO} /{ }^{13} \mathrm{CO} \mathrm{J}=3-2$ line ratio in one of the molecular clouds in IC 10 indicates that the gas must have a rather high kinetic temperature of about $100 \mathrm{~K}$. In IC 10 we observe structures on a variety of size scales that all appear to be gravitationally bound. This effect may help explain the rather high star formation rate in IC 10 . Subject headings: galaxies: irregular — galaxies: individual (IC 10, NGC 6822) - galaxies: ISM -
\end{abstract} ISM: molecules - Local Group

\section{INTRODUCTION}

Dwarf irregular galaxies are rather simple systems, free from the complicated dynamics that accompany bars or spiral density waves. This simplicity makes them useful for studying the interactions between star formation and the interstellar medium (ISM). Temperature, density, and abundance may all affect the star formation process, and thus, determining the physical conditions inside molecular clouds is important for understanding how the properties of the molecular gas affect the type and amount of stars formed. Unfortunately, extragalactic molecular clouds have low filling factors even within beams as small as $15^{\prime \prime}$, so their true temperatures cannot be deduced from the observed peak temperature of the spectral line. Instead, observations of many rotational transitions of $\mathrm{CO}$ and its isotopomers can be combined with radiative transfer models to constrain the physical conditions inside these clouds. As it is necessary to observe some of the rarer isotopomers, such as ${ }^{13} \mathrm{CO}$, these techniques have been primarily applied to starburst galaxies (e.g., Tilanus et al. 1991).

Relatively few dwarf galaxies have been detected in $\mathrm{CO}$, and the lines that are observed are much weaker than those found in spiral galaxies (Combes 1986; Tacconi \& Young 1987; Arnault et al. 1988). The weakness of these lines is likely due to a lower $\mathrm{CO}$ emissivity per unit mass of gas relative to spiral galaxies, which is caused by the low metallicity of most irregular galaxies $[12+\log (\mathrm{O} / \mathrm{H})=8.16$ for IC $10,8.20$ for NGC 6822; Lequeux et al. 1979]. IC 10 is one of the few dwarf galaxies in which strong $\mathrm{CO}$ lines have been detected (Becker 1990), with line strengths that are greater than those of the LMC, SMC, and NGC 6822 (e.g., Israel et al. 1993; Wilson 1994; Israel 1997). The simplest explanation of these strong $\mathrm{CO}$ lines is that IC 10 contains large amounts of molecular gas (Wilson \& Reid 1991). Unfortunately, except for the strong CO lines, there is no additional evidence to support this explanation. In fact, IC 10 has a rather low gas-to-dust ratio for a dwarf irregular galaxy (Schmidt \& Boller 1993). A more likely answer lies in the star forming activity. Massey \& Armandroff (1995) found that IC 10 has a global surface density of Wolf-Rayet stars 3 times as high as any other Local Group galaxy, comparable to the surface density found within active star forming regions of M33. This high concentration of WolfRayet stars reinforces the idea that IC 10 is undergoing a rather strong burst of star formation. It has been shown both observationally and theoretically that $\mathrm{CO}$ emission depends on the conditions in the molecular clouds. Maloney \& Black (1988) find that the ratio of CO intensity to excitation temperature is constant for ensembles of identical clouds. Hence, higher temperatures will result in brighter $\mathrm{CO}$ emission. The high star formation rates could increase the temperature of the molecular gas and more readily excite the $\mathrm{CO}$ molecules. Such a process would be able to produce the strong CO lines observed in IC 10.

In this paper we study the physical properties of molecular clouds in environments with different amounts of star forming activity located in two Local Group dwarf irregular galaxies, IC 10 and NGC 6822. IC 10 appears optically as a patchy nebulous region extending approximately $3^{\prime} \times 4^{\prime}$. It has been classified as a dwarf irregular of the Magellanic type (IBm; de Vaucouleurs \& Ables 1965). It is rather close to the Galactic plane $\left(b \sim-3^{\circ}\right)$, which indicates that there is heavy obscuration at visual wavelengths. It is considered a Local Group member based on its distance of $0.82 \mathrm{Mpc}$ (Wilson et al. 1996). The bright H II regions (e.g., Sandage \& Tammann 1974) indicate that IC 10 is undergoing massive star formation. NGC 6822 is also a dwarf irregular galaxy relatively close to the Galactic plane $\left(b \sim-18^{\circ}\right)$. It has a barlike appearance extending approximately $10^{\prime} \times 20^{\prime}$ north-south with a prominent "wing" located to the 
southeast. Its distance of $0.49 \mathrm{Mpc}$ (McGonegal et al. 1983) also places it in the Local Group. In $\S 2$ we discuss the observations and the data reduction process. In $\S 3$ we discuss the results found for the individual molecular clouds in IC 10 and NGC 6822 based on the line ratios and models. We also compare masses obtained from flux measurements with virial masses and discuss the validity of the assumptions made to aid in the interpretation of the data. In $\S 4$ we discuss the implications of these new data and the physical conditions of the molecular clouds for the CO-to$\mathrm{H}_{2}$ conversion factor. The paper is summarized in $\S 5$.

\section{OBSERVATIONS AND DATA REDUCTION}

Four regions centered on giant molecular clouds in IC 10 were observed using the James Clerk Maxwell Telescope (JCMT) over the period of 1994 October 20-24. The regions were selected from both interferometric CO $J=1-0$ surveys (Wilson \& Reid 1991; Wilson 1995) and a singledish ${ }^{12} \mathrm{CO} J=2-1$ and $J=1-0$ survey (Becker 1990). All four of the regions were observed in ${ }^{12} \mathrm{CO} J=2-1$, with follow-up observations for two of these regions (containing clouds IC 10: MC 1/2 \& IC 10: MC 6/7/8) in ${ }^{12} \mathrm{CO} J=3-2$ and ${ }^{13} \mathrm{CO} J=2-1$. A final ${ }^{13} \mathrm{CO} J=3-2$ observation was obtained at a single position located at the peak of the ${ }^{13} \mathrm{CO} J=2-1$ emission in IC10:MC $1 / 2$. The half-power beamwidth of the JCMT is $22^{\prime \prime}$ at $230 \mathrm{GHz}\left({ }^{12} \mathrm{CO} \mathrm{J}=2-1\right)$ and $15^{\prime \prime}$ at $345 \mathrm{GHz}\left({ }^{12} \mathrm{CO} J=3-2\right)$. Data were obtained on a five-point cross offset by $11^{\prime \prime}$ for the $J=2-1$ data and $8^{\prime \prime}$ for the $J=3-2$ data. One giant molecular cloud in NGC 6822 was observed during service observing at the JCMT on the nights of 1996 May $5\left({ }^{12} \mathrm{CO} J=3-2\right)$ and 1994 June $8\left({ }^{12} \mathrm{CO} J=2-1\right)$. The cloud was observed at a single position in the $J=2-1$ transition and at a five-point cross offset by $8^{\prime \prime}$ in the $J=3-2$ transition. All observations were obtained using the Dwingeloo Autocorrelation Spectrometer.

The data were reduced using the Bell Labs data reduction package COMB. The data were binned to $1.5 \mathrm{~km} \mathrm{~s}^{-1}$ resolution and first to seventh degree baselines were removed. The lines were quite narrow (less than $30 \mathrm{~km} \mathrm{~s}^{-1}$ ) and the spectrometer bandwidth was at least $450 \mathrm{~km} \mathrm{~s}^{-1}$, so the removal of rather high degree baselines for some spectra do not introduce significant errors into the final line intensities. Third and seventh degree baseline removal on the
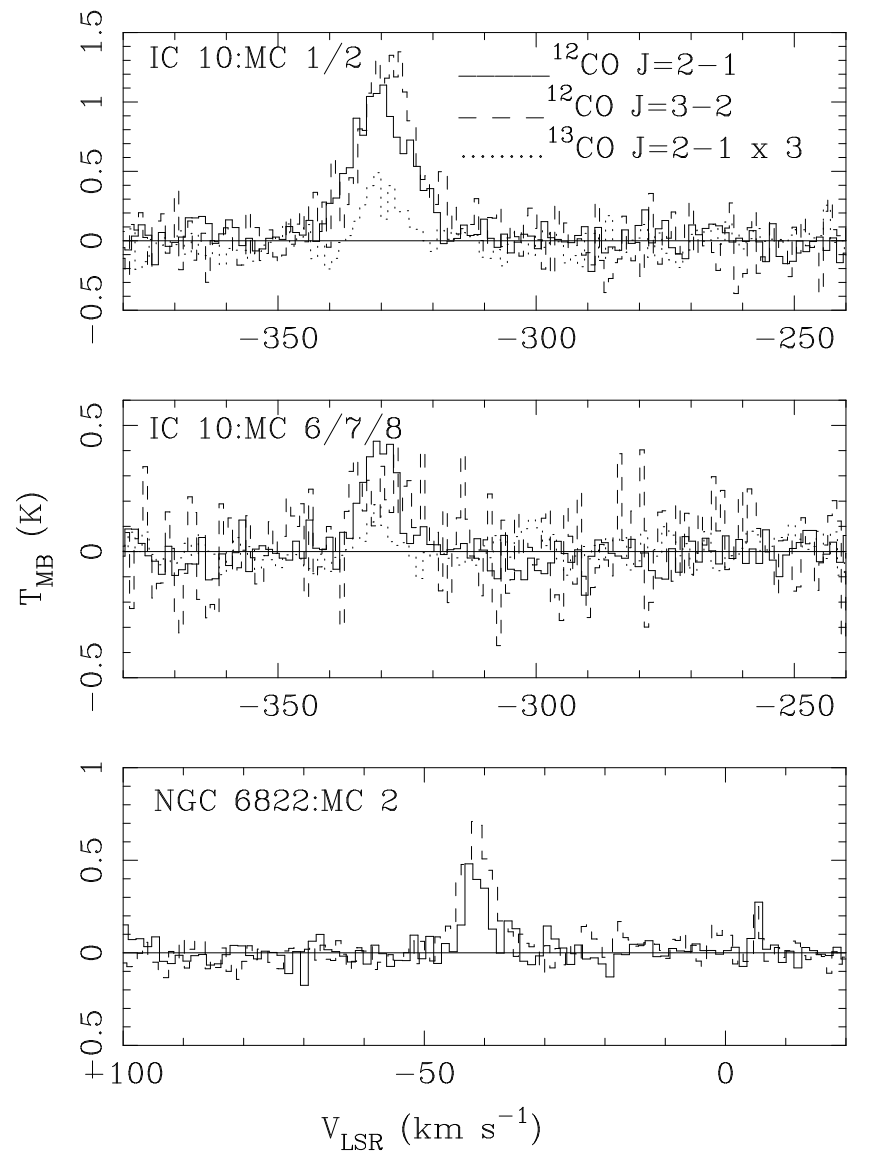

FIG. 1.-The ${ }^{12} \mathrm{CO} J=2-1$ (solid line), ${ }^{13} \mathrm{CO} J=2-1$ (dotted line), and ${ }^{12} \mathrm{CO} J=3-2$ (dashed line) spectra observed in three regions in IC 10 and NGC 6822 . The $J=3-2$ data have been convolved to match the $22^{\prime \prime}$ beam of the $J=2-1$ data. The ${ }^{13} \mathrm{CO} J=2-1$ line has been scaled up by a factor of 3 .

same data yielded line parameters that agreed to within $1 \sigma$. The ${ }^{12} \mathrm{CO} J=3-2$ data were then convolved to simulate a $22^{\prime \prime}$ beam. The spectra for each cloud are shown in Figures 1 and 2. The data were then binned to the same resolution and shifted to the same $V_{\mathrm{LSR}}$ (when necessary). This allowed the calculation of line ratios for each channel within the spectral line. The ratios for each channel were averaged to

TABLE 1

CO Line Ratios of Molecular Clouds in IC 10 and NGC 6822

\begin{tabular}{|c|c|c|c|c|c|c|}
\hline Cloud $^{\mathrm{a}}$ & $\alpha(1950)$ & $\delta(1950)$ & $\frac{{ }^{12} \mathrm{CO} J=2-1}{{ }^{13} \mathrm{CO} J=2-1}$ & $\frac{{ }^{12} \mathrm{CO} J=3-2}{{ }^{12} \mathrm{CO} J=2-1}$ & $\frac{{ }^{12} \mathrm{CO} J=3-2}{{ }^{13} \mathrm{CO} J=3-2}$ & $\frac{{ }^{12} \mathrm{CO} J=2-1}{{ }^{12} \mathrm{CO} J=1-0}$ \\
\hline IC $10: \mathrm{MC} 1 / 2$ & 001746.2 & +590028 & $8.2 \pm 0.6$ & $0.90 \pm 0.11$ & $5.3 \pm 0.4$ & $0.64 \pm 0.05$ \\
\hline IC $10: R 2 \ldots \ldots \ldots$ & 001734.7 & +590205 & $\ldots$ & $\ldots$ & $\ldots$ & $1.18 \pm 0.1$ \\
\hline IC $10:$ MC $4 \ldots \ldots \ldots \ldots$ & 001737.5 & +590408 & $\ldots$ & $\ldots$ & $\ldots$ & $0.65 \pm 0.06$ \\
\hline IC $10:$ MC $6 / 7 / 8 \ldots \ldots$. & 001740.1 & +590438 & $8.3 \pm 1.4$ & $0.66 \pm 0.11$ & $\ldots$ & $0.50 \pm 0.06$ \\
\hline NGC 6822:MC $2 \ldots \ldots$ & 194203.3 & -145027 & $\ldots$ & $1.54 \pm 0.08$ & $\ldots$ & \\
\hline M33 average ${ }^{\mathrm{b}} \ldots \ldots \ldots$ & $\ldots$ & $\ldots$ & $7.2 \pm 1.1$ & $0.69 \pm 0.15$ & $\ldots$ & $0.67 \pm 0.19$ \\
\hline
\end{tabular}

Notes.-Units of right ascension are hours, minutes, and seconds, and units of declination are degrees, arcminutes, and arcseconds. All data obtained at JCMT $15 \mathrm{~m}$, except for the ${ }^{12} \mathrm{CO} J=1-0$ data, which are taken from Becker 1990. All line ratios refer to a $22^{\prime \prime}$ beam, except for the ${ }^{12} \mathrm{CO} /{ }^{13} \mathrm{CO} J=3-2$ ratio, which is for a $15^{\prime \prime}$ beam. The line ratios are the average of all channels with a $\mathrm{S} / \mathrm{N}$ greater than 2 (see Fig. 3), and the uncertainties are the standard deviation of the mean.

${ }^{a}$ Cloud designations from Wilson \& Reid 1991; Wilson 1994, 1995; except for R2 (Becker 1990).

${ }^{\mathrm{b}}$ From Wilson et al. 1997; Thornley \& Wilson 1994. 


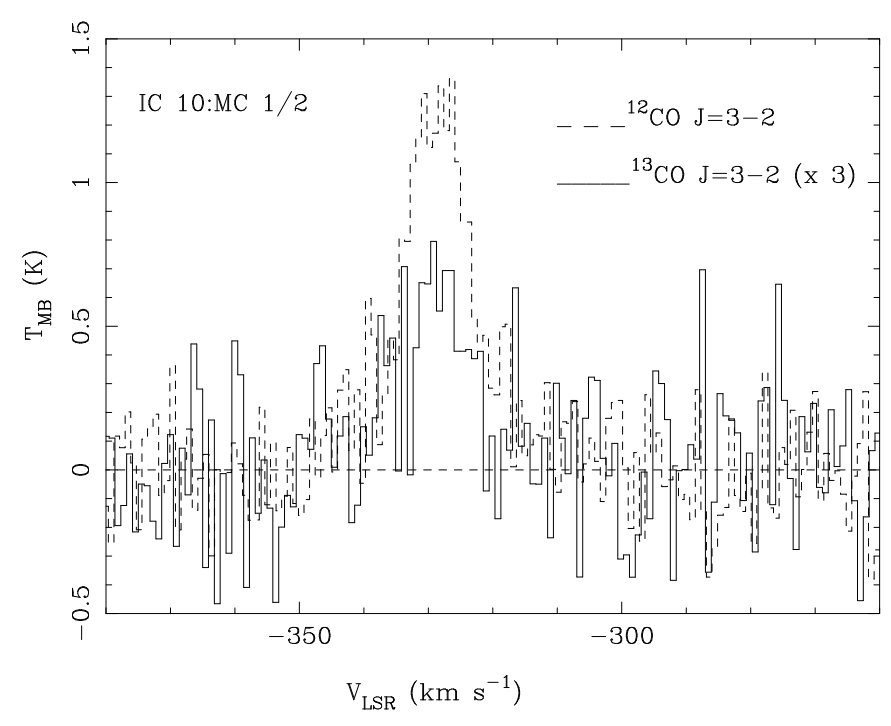

FIG. 2.-The ${ }^{12} \mathrm{CO} J=3-2$ (dashed line) and ${ }^{13} \mathrm{CO} J=3-2$ (solid line) spectra observed toward the region MC $1 / 2$ in IC 10 . The spectra were obtained with a $15^{\prime \prime}$ beam. The ${ }^{13} \mathrm{CO} J=3-2$ spectrum is scaled up by a factor of 3 .

obtain the final line ratios. Only those channels with a signal-to-noise ratio of at least 2 in the line ratio were used in the calculation. This method helps reduce errors introduced by the existence of poor baselines or weak signals. The uncertainty in the value for the line ratio is taken as the standard deviation of the mean. As a final step, the line ratios were scaled to the main beam temperature scale using the appropriate values for $\eta_{\mathrm{MB}}$ (see below). A summary of the line ratios is given in Table 1, and a channel by channel histogram of the ratios is shown in Figure 3.

The calibration of the IC 10 data was monitored by frequently observing both planets and spectral line calibrators. Unfortunately, it was later determined that the beam of the telescope was decidedly noncircular during 1994 October (see JCMT Newsletter No. 4, March 1995), enough to affect our calibration. Although the spectral line calibrators showed considerable scatter, with individual measurements differing by as much as $\pm 40 \%$ from standard spectra, observations of a given source were repeatable to within $\sim 5 \%$. The wide scatter of the spectral line calibrators may be understood given the large ellipticity of the beam and the range in diameter of the calibrators, from compact carbon stars to more extended emission in $\mathrm{H}$ II regions. Depending on the size of the source, the measured line strength may be larger or smaller with the elliptical beam compared to that measured with a circular beam. The main beam efficiencies determined using Saturn were $0.60 \pm 0.02$ at $220 / 230 \mathrm{GHz}$ and $0.36 \pm 0.01$ at $345 \mathrm{GHz}$. Both efficiencies are substantially lower than normal values. Since Saturn had a diameter of $18^{\prime \prime}$ during these observations, it provides a reasonable match to the size of the emission regions in IC 10. This assumption was confirmed by observing the molecular cloud MC 20 in M33, for which observations obtained with the JCMT in a more normal state were available; the ${ }^{12} \mathrm{CO} J=2-1$ line from this source was indeed low by about $20 \%$. Thus, we adopt these observed efficiencies to convert our data to the main beam temperature scale. For the NGC 6822 data (taken at a different dates under normal calibration conditions), observations of CRL 2688 agreed
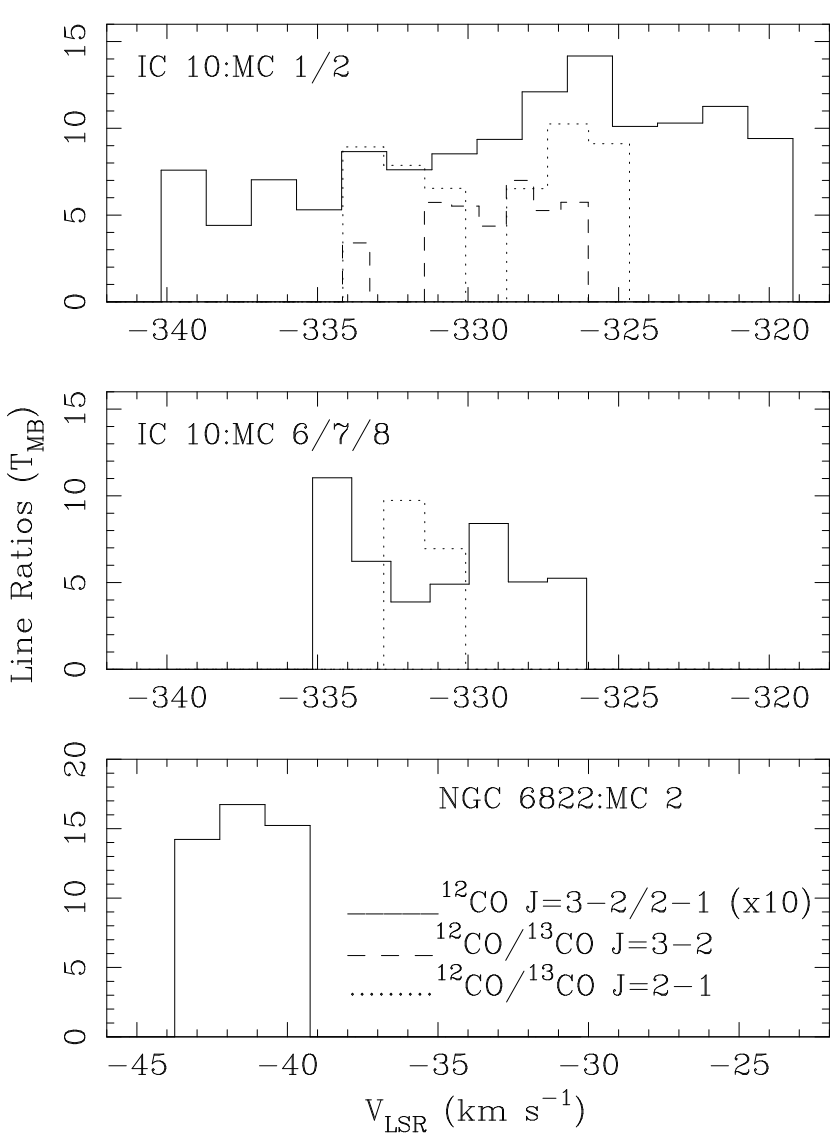

FIG. 3.-Channel by channel line ratios (in $T_{\mathrm{MB}}$ ) for the three regions observed. The solid line represents the ${ }^{12} \mathrm{CO} J=3-2 / J=2-1$ line ratio (scaled up by a factor of 10), the dashed line represents the ${ }^{12} \mathrm{CO} /{ }^{13} \mathrm{CO}$ $J=3-2$ line ratio, and the dotted line represents the ${ }^{12} \mathrm{CO} /{ }^{13} \mathrm{CO} J=2-1$ line ratio. Only those channels with $\mathrm{S} / \mathrm{N}$ greater than 2 are shown.

with the published values, and so we adopt the normal main beam efficiencies from the JCMT Users Guide of 0.72 at 230 $\mathrm{GHz}$ and 0.58 at $345 \mathrm{GHz}$.

Since the line strengths we are comparing are measured with the same beam diameter, using the $T_{R}^{*}$ temperature scale would ensure that our observed line ratios are equal to the true radiation temperature ratios. However, conversion to $T_{R}^{*}$ from $T_{\mathrm{A}}^{*}$ requires knowledge of the forward scattering and spillover $\left(\eta_{\mathrm{FSS}}\right)$, which is difficult to measure and was not attempted during the observing run. As a result, we do not know how to correct the published values of $\eta_{\text {Fss }}$ to take into account the calibration problems discussed above. On the other hand, we do have good values for the main beam efficiencies, so an accurate conversion to main beam temperature $\left(T_{\mathrm{MB}}\right)$ is possible. Using the $T_{\mathrm{MB}}$ scale instead of the $T_{R}^{*}$ scale only changes the ${ }^{12} \mathrm{CO} J=3-2 / J=2-1$ line ratio by $\sim 5 \%$ under normal calibration conditions. For this reason we will use the main beam temperature scale for the line ratios throughout this paper.

\section{MOLECULAR CLOUD PROPERTIES}

\section{1. $M C 1 / 2$ and $M C 6 / 7 / 8$ in IC 10}

The clouds MC $1 / 2$ are located $60^{\prime \prime}(240 \mathrm{pc}$ at a distance of $0.82 \mathrm{Mpc}$ ) from the center of the brightest $\mathrm{H}$ II region in IC 10 (No. 111; Hodge \& Lee 1990; Shostak \& Skillman 1989). This $\mathrm{H}$ II region has an $\mathrm{H} \alpha$ luminosity of $4 \times 10^{38}$ 
ergs $\mathrm{s}^{-1}$. Conversely, MC 6/7/8 is not located near a large $\mathrm{H}$ II region; it is $86^{\prime \prime}(340 \mathrm{pc})$ away from the edge of even the nearest moderate-sized one (No. 50, $\mathrm{H} \alpha$ luminosity of $\sim 10^{37}$ ergs s$^{-1}$; Hodge \& Lee 1990). For these two regions (MC $1 / 2$ and $\mathrm{MC} 6 / 7 / 8$ ) the ${ }^{12} \mathrm{CO} /{ }^{13} \mathrm{CO} J=2-1$ ratios agree very well, which suggests similar ${ }^{12} \mathrm{CO} /{ }^{13} \mathrm{CO}$ abundances in these clouds. Although the ${ }^{12} \mathrm{CO} J=3-2 / J=2-$ 1 ratios also agree within the large uncertainties, the larger ratio of 0.90 is found for the cloud nearer the bright $\mathrm{H}$ II region, while the lower ratio of 0.66 is found in the more quiescent cloud.

Wilson, Walker, \& Thornley (1997) found that the giant molecular cloud NGC 604-2, located in the very intense star formation environment of the giant $\mathrm{H}$ II region NGC 604 in M33, had a ${ }^{12} \mathrm{CO} J=3-2 / J=2-1$ ratio that was higher than average for M33 $(1.07 \pm 0.17$, compared to $0.64 \pm 0.07$ for clouds without optical $\mathrm{H}$ II regions). They also found that a cloud located only 120 pc (31") away from NGC 604 did not show an increase in the ${ }^{12} \mathrm{CO} J=3-2 / J=2-1$ ratio. In IC $10, \mathrm{MC} 1 / 2$ does exhibit an enhanced ${ }^{12} \mathrm{CO}$ $J=3-2 / J=2-1$ line ratio, even though it is farther away from the weaker H II region No. 111. This result suggests that if a similar line ratio enhancement is taking place as in M33, the ISM in the metal-poor dwarf irregular galaxy IC 10 must be more porous to UV radiation. This effect could allow the line ratio enhancement to occur at greater distances from weaker $\mathrm{H}$ II regions than is possible in the higher metallicity spiral galaxy M33 $[12+\log (\mathrm{O} / \mathrm{H})=$ 8.48 in NGC 604; Vilchez et al. 1988].

The IC 10 and the NGC 6822 data (see $\S 3.2$ ) support the conclusions of Wilson et al. (1997) that the ${ }^{12} \mathrm{CO} J=3-2 /$ $J=2-1$ line ratio increases toward the centers of bright $\mathrm{H}$ II regions. The higher transitions can be more readily excited by higher temperatures or higher densities, either of which will produce higher line ratios. It is believed that higher temperatures are present near star-forming regions, but without accurate densities for these clouds, we cannot assess the extent to which both of these factors contribute to the elevated line ratios. Independent temperature information on these clouds (e.g., from the new bolometer array SCUBA on the JCMT) should yield a clearer picture of the processes at work.

For the clouds for which we have observed more than one line ratio, we can use models to help constrain the physical conditions of the clouds. We will use the large velocity gradient (LVG) model, which assumes that the clouds in the beam have internal velocities large enough to Doppler shift the $\mathrm{CO}$ emission from one part of the cloud sufficiently to prevent reabsorption by other CO molecules in other parts of the cloud (Scoville \& Solomon 1974). We used the LVG code RAD, written by L. Mundy and implemented as part of the MIRIAD data reduction package. Model grids were run for kinetic temperatures $T_{\mathrm{K}}=10,15$, $20,30,50,100,200,300 \mathrm{~K}$, abundance ratios $\left[{ }^{12} \mathrm{CO}\right] /$ $\left[{ }^{13} \mathrm{CO}\right]=30,50,70$, covering a range of densities of $\log$ $\left[n_{\mathrm{H}_{2}}\left(\mathrm{~cm}^{-3}\right)\right]=1-6$ and ${ }^{12} \mathrm{CO}$ column densities per unit velocity of $\log \left[N\left({ }^{12} \mathrm{CO}\right) / \Delta v\left(\mathrm{~cm}^{-2} \mathrm{~km}^{-1} \mathrm{~s}\right)\right]=15-20$. To calculate the ${ }^{12} \mathrm{CO}$ column densities from $N / \Delta v$, we use the FWHM velocity.

Figure 4 shows one of the two solutions found for the line ratios of IC 10: $\mathrm{MC} 1 / 2$. The three ratios obtained using the JCMT $\left({ }^{12} \mathrm{CO} /{ }^{13} \mathrm{CO} J=3-2,{ }^{12} \mathrm{CO} J=3-2 / J=2-1\right.$ and $\left.{ }^{12} \mathrm{CO} /{ }^{13} \mathrm{CO} J=2-1\right)$ are shown as bands that indicate the $1 \sigma$ upper and lower limits of the line ratio. Solutions are

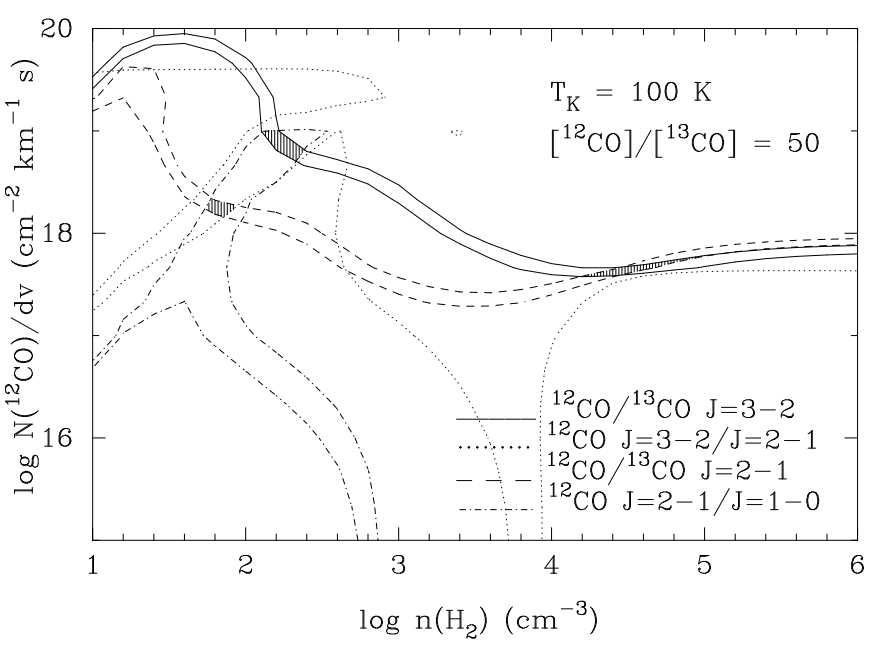

FIG. 4.- Solutions for the density and column density for the region IC 10:MC $1 / 2$ using a large-velocity gradient code. This figure shows the solution for the three JCMT line ratios at a kinetic temperature of $100 \mathrm{~K}$ and a $\left[{ }^{12} \mathrm{CO}\right] /\left[{ }^{13} \mathrm{CO}\right]$ abundance of 50 . The solid lines indicate the $1 \sigma$ limits of the ${ }^{12} \mathrm{CO} /{ }^{13} \mathrm{CO} J=3-2$ line ratio of $5.3 \pm 0.4$. The dotted lines represent the ${ }^{12} \mathrm{CO} J=3-2 / J=2-1$ line ratio of $0.9 \pm 0.11$, and the dashed lines represent the ${ }^{12} \mathrm{CO} /{ }^{13} \mathrm{CO} J=2-1$ line ratio of $8.2 \pm 0.6$. Also included is the ${ }^{12} \mathrm{CO} J=2-1 / J=1-0$ line ratio of $0.64 \pm 0.05(J=1-0$ data from IRAM; Becker 1990), shown as the dot-dashed lines. Solutions are indicated by the shaded regions where the line ratios overlap. In this case there are three separate regions where three of the four line ratios overlap that may indicate two or more distinct emission regions within the clouds.

indicated by the locations where the regions of the different ratio boundaries overlap. Of the 24 possible models, this only occurred twice for the JCMT line ratios of IC 10:MC $1 / 2$, at $T_{\mathrm{K}}=100 \mathrm{~K}$ and for the abundances $\left[{ }^{12} \mathrm{CO}\right] /$ $\left[{ }^{13} \mathrm{CO}\right]=50$ and 70 . Both cases indicate a density of $10^{4}-10^{5} \mathrm{~cm}^{-3}$ and column densities per unit velocity of about $10^{17.7} \mathrm{~cm}^{-2}\left(\mathrm{~km} \mathrm{~s}^{-1}\right)^{-1}$. The average FWHM of the emission lines in IC 10:MC $1 / 2$ is $12.2 \pm 1.4 \mathrm{~km} \mathrm{~s}^{-1}$, which gives a true column density of $6 \times 10^{18} \mathrm{~cm}^{-2}$.

We have also calculated the ${ }^{12} \mathrm{CO} J=2-1 / J=1-0$ ratio by combining our JCMT ${ }^{12} \mathrm{CO} J=2-1$ data (22" beam) and the IRAM $30 \mathrm{~m}$ telescope ${ }^{12} \mathrm{CO} J=1-0$ data $\left(21^{\prime \prime}\right.$ beam) obtained by Becker (1990). The similarity in the beam sizes at these two frequencies allows a straightforward combination of the data sets without the use of convolution algorithms. In an attempt to constrain the abundance better, we then included the ${ }^{12} \mathrm{CO} J=2-1 / J=1-0$ line ratio in the LVG model. However, instead of overlapping with one of the other two existing solutions, the ${ }^{12} \mathrm{CO}$ $J=2-1 / J=1-0$ line ratio provided two additional solutions for each abundance at lower density $\left(n\left(\mathrm{H}_{2}\right)=\right.$ $10^{1.5}-10^{2.5} \mathrm{~cm}^{-3}$ ). There is no region where all four line ratios overlap. This result may indicate the existence of separate high- and low-density regions that contribute to the emission. The high-density region dominates the upper $J$ emission, while the lower $J$ emission would originate predominantly from the lower density region (as would be predicted by the Boltzmann equation). We know that Galactic molecular clouds are clumpy (e.g., Stutzki \& Güsten 1990), and there is every reason to believe that extragalactic molecular clouds are clumpy as well. If this is the case, we can perhaps interpret the two distinct emitting regions as representing the high-density clumps and the less dense 
interclump regions. Unfortunately, the lack of data with resolution better than 7" or 28 pc (Wilson \& Reid 1991; Ohta et al. 1992; Wilson 1995) precludes further discussion of the degree of substructure within molecular clouds in IC 10.

With only two line ratios $\left({ }^{12} \mathrm{CO} J=3-2 / J=2-1\right.$ and $\left.{ }^{12} \mathrm{CO} /{ }^{13} \mathrm{CO} J=2-1\right)$, it is not possible to put very strong constraints on the physical conditions in IC 10 MC 6/7/8 without making some assumptions. For example, if we assume a moderate temperature of $20 \mathrm{~K}$ and a $\left[{ }^{12} \mathrm{CO}\right] /$ $\left[{ }^{13} \mathrm{CO}\right]$ abundance of 50 (as found for IC $10: \mathrm{MC} 1 / 2$ ), we can estimate upper and lower limits for the densities in the cloud. The results of this model are shown in Figure 5. There is a rather broad range of allowed values for density $\left(10^{1.6}-10^{4} \mathrm{~cm}^{-3}\right)$ and for ${ }^{12} \mathrm{CO}$ column densities per unit velocity $\left(10^{16.5}-10^{18.5} \mathrm{~cm}^{-2}\left(\mathrm{~km} \mathrm{~s}^{-1}\right)^{-1}\right)$. The FWHM of the emission lines in $\mathrm{MC} 6 / 7 / 8$ is $7.1 \pm 1.6 \mathrm{~km} \mathrm{~s}^{-1}$, which gives true column densities ranging from $2 \times 10^{17}$ to $2 \times 10^{19} \mathrm{~cm}^{-2}$. If we assume a $\left[{ }^{12} \mathrm{CO}\right] /\left[{ }^{13} \mathrm{CO}\right]$ abundance of 70 (as is also allowed by the more complete IC 10:MC $1 / 2$ model) the only change is that the lower limit on column density increases from $2 \times 10^{17}$ to $7 \times 10^{17} \mathrm{~cm}^{-2}$, narrowing the allowed range of column density slightly. The range in density and the upper limit in column density remain essentially the same. Adopting a higher kinetic temperature of $30 \mathrm{~K}$ results only in a slightly lower upper limit on $\mathrm{H}_{2}$ density $\left(10^{3.6} \mathrm{~cm}^{-3}\right.$ instead of $\left.10^{4} \mathrm{~cm}^{-3}\right)$. Adopting a cooler kinetic temperature of $10 \mathrm{~K}$ indicates that $\mathrm{H}_{2}$ densities greater than $10^{4} \mathrm{~cm}^{-3}$ are required to produce the observed ${ }^{12} \mathrm{CO} \quad J=3-2 / J=2-1$ and ${ }^{12} \mathrm{CO} /{ }^{13} \mathrm{CO} \quad J=2-1$ line ratios. As in IC 10: MC $1 / 2$, the ${ }^{12} \mathrm{CO} J=2-1 / J=1-0$ line ratio for MC 6/7/8 does not overlap with the JCMT line ratios, which again suggests we may be seeing a combination of high- and low-density gas in this region.

\section{2. $M C 2$ in NGC 6822}

The molecular cloud MC 2 in NGC 6822 is located almost in the very center (within $1^{\prime \prime}$ ) of the brightest $\mathrm{H}$ II

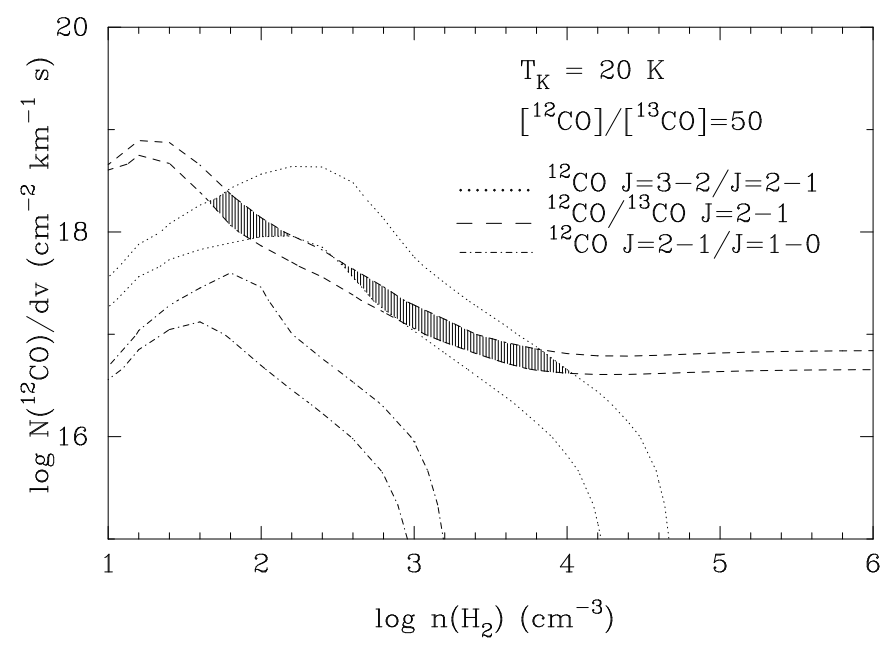

FIG. 5.-Typical LVG solution for the region MC 6/7/8 in IC 10 for a kinetic temperature of $20 \mathrm{~K}$ and a $\left[{ }^{12} \mathrm{CO}\right] /\left[{ }^{13} \mathrm{CO}\right]$ abundance of 50 . The dotted lines represent the $1 \sigma$ limits of the ${ }^{12} \mathrm{CO} J=3-2 / J=2-1$ line ratio of $0.66 \pm 0.11$, while the dashed lines indicate the ${ }^{12} \mathrm{CO} /{ }^{13} \mathrm{CO} J=2-1$ line ratio of $8.3 \pm 1.4$. The shaded region indicates the solutions for the JCMT data. Also included is the ${ }^{12} \mathrm{CO} J=2-1 / J=1-0$ line ratio of $0.50 \pm 0.06$ $\left({ }^{12} \mathrm{CO} J=1-0\right.$ data from Becker 1990$)$. The ${ }^{12} \mathrm{CO} J=2-1 / J=1-0$ line ratio does not overlap with either of the JCMT line ratios. region in NGC 6822. Discovered by Hubble (1925) and designated H v by Hodge, Kennicutt, \& Lee (1988), this H II region has an $\mathrm{H} \alpha$ luminosity of $4 \times 10^{38} \mathrm{ergs} \mathrm{s}^{-1}$ (Hodge, Lee, \& Kennicutt 1989). The ${ }^{12} \mathrm{CO} J=3-2 / J=2-1$ ratio $(1.40 \pm 0.07)$ is significantly higher than either of the IC 10 line ratios. The $\mathrm{H}$ II region $\mathrm{H} \mathrm{v}$ is weaker than the giant $\mathrm{H}$ II region NGC 604 in M33 $\left(\mathrm{H} \alpha\right.$ luminosity of $5 \times 10^{39} \mathrm{ergs}$ $\mathrm{s}^{-1}$; Kennicutt 1988), yet we see a much higher ${ }^{12} \mathrm{CO}$ $J=3-2 / J=2-1$ line ratio in NGC 6822:MC 2. Again, as in IC 10, we see that in the low-metallicity environment of NGC 6822, molecular clouds associated with $\mathrm{H}$ II regions can attain line ratios higher than in higher metallicity galaxies such as M33.

High $J$ transitions can be readily excited by high temperatures, high densities, or some combination of the two. We would expect the temperature of molecular clouds near $\mathrm{H}$ II regions to be higher than those unassociated with $\mathrm{H}$ II regions. These higher temperatures would more readily excite the higher $J$ transitions even in relatively low density regions. In the low density hot gas there will be less $\mathrm{CO}$ in the lowest rotational levels, and hence a lower probability of being reabsorbed (i.e., lower optical depth). Without separate constraints on the density, it is difficult to assess the role that density plays in producing this high line ratio. However, it can be shown from the virial theorem that for clouds with a relatively constant surface pressure, the product of density and temperature $(\rho T)$ is approximately constant (e.g., equation [2.8d], McLaughlin \& Pudritz 1996). This result implies that for virialized clouds, an increase in temperature results in a decrease in density. It is likely that this cloud in NGC 6822 is in virial equilibrium (Wilson 1994; § 4). It also seems likely in the case of MC 2 that the temperature should be higher than in typical interstellar molecular clouds because of its close proximity to the $\mathrm{OB}$ stars generating the large $\mathrm{H}$ II region. We therefore conclude that in MC 2 the high line ratio is most likely the result of the high temperatures due to the proximity of the bright $\mathrm{H}$ in region.

With only one line ratio, a detailed LVG analysis is not possible. If we assume that the cloud is in local thermodynamic equilibrium (LTE), we would expect line ratios no greater than unity for optically thick gas, while optically thin gas can produce much larger ratios (e.g., Walker 1991). The observed line ratio indicates that the ${ }^{12} \mathrm{CO}$ gas is optically thin in MC 2. CO is the most abundant molecule after $\mathrm{H}_{2}$ and is generally found to be optically thick in giant molecular clouds. Optically thin ${ }^{12} \mathrm{CO}$ is quite rare, and likely the result of special physical conditions inside an $\mathrm{H} \mathrm{II}$ region or possibly in starburst galaxies.

For gas to be molecular, it must be shielded by dust or have a high enough column density to be self-shielding. For molecular gas to collapse into stars, it is believed that the column density must exceed some critical value (e.g., Mouschovias \& Spitzer 1976; Herbig \& Terndrup 1986). These values of column density are comparable and are believed to be on the order of $10^{22} \mathrm{~cm}^{-2}$ for the predominantly molecular clouds in the Galaxy (Solomon et al. 1987; Elmegreen 1989; McLaughlin \& Pudritz 1996). In starbursts, the gas density may vary depending on how long ago the burst of star formation began. Initially, the collapse of the molecular clouds could give a higher than average gas density. The density should decrease as the gas is used up, new hot stars heat and expand the gas, and stellar winds blow gas out of the galaxy. This heating and expansion of 
the gas in a molecular cloud will decrease the density while leaving the column density unchanged (McLaughlin \& Pudritz 1996). However, increasing the temperature excites more molecules to higher rotational levels, which decreases the opacity in the lower rotational levels. We should then expect to see optically thick gas in gravitationally subcritical molecular clouds, unless the clouds are heated sufficiently by external sources, such as the active star forming regions we see in NGC 6822.

This heating effect may be amplified in the lowmetallicity environment of NGC 6822. Lower metallicity suggests a lower dust content, which would reduce the amount of shielding by dust from UV radiation from the star forming regions. With this reduced shielding, the UV radiation is able to heat the molecular gas more efficiently than is possible in higher metallicity (higher dust content) environments, which could contribute to the reduced optical depth seen in this galaxy. We might also expect optically thin gas in regions where there is simply less molecular gas. There is evidence for a deficit of molecular gas in the weak CO line strengths of NGC 6822 . We thus attribute the low optical depth of the molecular gas in NGC 6822 to the low molecular gas content in this metal-poor environment. Our data are insufficient to sort out the extent to which each factor (namely low gas content and low metallicity) contributes to the low optical depth.

Optically thick gas (assuming LTE) can only produce line ratios $\leq 1$, whereas optically thin gas can produce ${ }^{12} \mathrm{CO}$ $J=3-2 / J=2-1$ line ratios of $\gtrsim 2.5$ for temperatures greater than $100 \mathrm{~K}$ (Walker 1991). It is possible that only a fraction of the gas in MC 2 is optically thin. For example, the cloud could be a mixture of $10 \%$ optically thin gas and $90 \%$ optically thick gas. Assuming the optically thin CO emission is not blocked by the optically thick emission, we would then expect our line ratios to be on the order of $1(0.9)+2.5(0.1)=1.15$ for a $100 \mathrm{~K}$ cloud. A rigorous calculation of the effect of mixtures of optically thin and thick gas is complicated and beyond the scope of this paper. However, this simple calculation suggests that MC 2 contains at least $\sim 25 \%$ optically thin gas. For simplicity, we will assume that the observed high ${ }^{12} \mathrm{CO} J=3-2 / J=2-1$ line ratio indicates that the entire cloud is optically thin.

With only one line ratio for MC $2\left({ }^{12} \mathrm{CO} J=3-2 / J=\right.$ $2-1)$, we can only place crude limits on the temperature and density. However, we do not need to make any assumptions about the abundance ratio, since our data do not include any ${ }^{13} \mathrm{CO}$ observations. Figure 6 shows a possible solution for a temperature of $100 \mathrm{~K}$. This temperature seems reasonable, considering MC 2 is located within a massive star forming region. If we assume a temperature any lower than $50 \mathrm{~K}$, we would require densities greater than $10^{6} \mathrm{~cm}^{-3}$ to achieve the observed line ratios. While this is not impossible, it seems more likely that this line ratio is a result of higher temperatures. For a temperature of $100 \mathrm{~K}$, we can put a lower limit of $10^{4.2} \mathrm{~cm}^{-3}$ on density and an upper limit of $10^{17} \mathrm{~cm}^{-2}\left(\mathrm{~km} \mathrm{~s}^{-1}\right)^{-1}$ on column density per unit velocity. The FWHM velocity of the emission lines in NGC 6822 is $5.4 \pm 0.9 \mathrm{~km} \mathrm{~s}^{-1}$, which implies a true upper limit on column density of $5 \times 10^{17} \mathrm{~cm}^{-2}$.

If we increase the temperature above $100 \mathrm{~K}$, we see that lower $\mathrm{H}_{2}$ densities are allowed by the models. The observed line ratios can be produced with $\mathrm{H}_{2}$ densities as low as 100 $\mathrm{cm}^{-3}$ with temperatures of $200 \mathrm{~K}$, but the $\mathrm{CO}$ column densities increase to greater than $5 \times 10^{18} \mathrm{~cm}^{-2}$. Assuming a

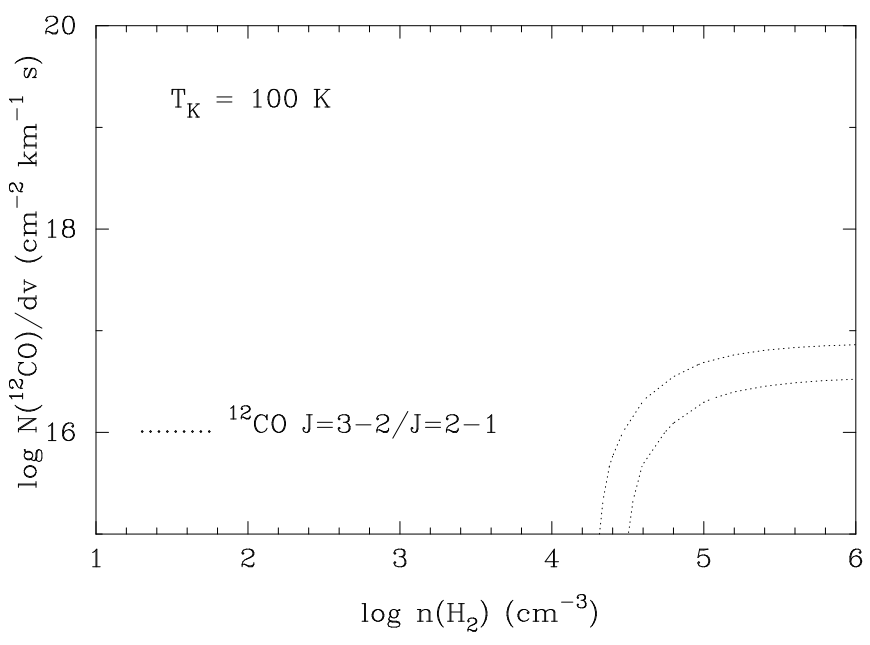

FIG. 6.-One possible temperature $(100 \mathrm{~K})$ solution for the region MC 2 in NGC 6822. The dotted lines show the $1 \sigma$ limits of the ${ }^{12} \mathrm{CO} J=3-2 /$ $J=2-1$ line ratio of $1.4 \pm 0.07$. With only a single line ratio, it is only possible to place lower limits on the temperature $(>50 \mathrm{~K})$ and density $\left(>10^{4} \mathrm{~cm}^{-3}\right)$.

number density ratio of $n(\mathrm{CO}) / n\left(\mathrm{H}_{2}\right)$ of $10^{-4}$ (e.g., Genzel 1992) we obtain an $\mathrm{H}_{2}$ column density of about $5 \times 10^{22}$ $\mathrm{cm}^{-2}$. This column density is uncomfortably close to the critical column density for molecular cloud collapse of $\sim 10^{22} \mathrm{~cm}^{-2}$. As it seems unlikely that we are fortunate enough to observe a cloud that is on the brink of collapse, we will use the above value of the $\mathrm{CO}$ column density of $5 \times 10^{17} \mathrm{~cm}^{-2}$ in further calculations.

\subsection{Molecular Masses}

The intensity of the $\mathrm{CO}$ emission can be related to the molecular mass of a cloud using the equation

$$
M_{\mathrm{mol}}=1.61 \times 10^{4}\left(\frac{\alpha}{\alpha_{\mathrm{Gal}}}\right)\left(\frac{115 \mathrm{GHz}}{v}\right)^{2} d_{\mathrm{Mpc}}^{2} \frac{S_{\mathrm{CO}}}{R} M_{\odot}
$$

(Wilson \& Scoville 1990; Wilson 1995), where $S_{\mathrm{CO}}$ is the ${ }^{12} \mathrm{CO} J=2-1$ flux in $\mathrm{Jy} \mathrm{km} \mathrm{s}{ }^{-1}, R$ is the ${ }^{12} \mathrm{CO} J=2-1 /$ $J=1-0$ line ratio, $v$ is the frequency of the emission (230 $\mathrm{GHz}$ for the $J=2-1$ transition), $d_{\mathrm{Mpc}}$ is the distance to the cloud in Mpc, $\alpha$ is the CO-to- $\mathrm{H}_{2}$ conversion factor for the galaxy, and $\alpha_{\mathrm{Gal}}$ is the Galactic value $\left[3 \pm 1 \times 10^{20} \mathrm{~cm}^{-2}\right.$ $\left(\mathrm{K} \mathrm{km} \mathrm{s}^{-1}\right)^{-1}$; Strong et al. 1988; Scoville \& Sanders 1987]. The IC $10{ }^{12} \mathrm{CO} J=2-1 / J=1-0$ line ratio was calculated by combining Becker's (1990) IRAM ${ }^{12} \mathrm{CO} J=1-0$ (21" beam) with our JCMT ${ }^{12} \mathrm{CO} J=2-1$ data (22" beam); see Table 1. For NGC 6822, a ${ }^{12} \mathrm{CO} J=2-1 / J=1-0$ ratio of 0.7 is assumed. The CO-to- $\mathrm{H}_{2}$ conversion factor $(\alpha)$ is a globally averaged property of the galaxy, hence there are uncertainties involved in its use in one specific region of the galaxy. Recent studies have suggested that the global CO-to- $\mathrm{H}_{2}$ conversion factor in IC 10 may be much higher than in our own Galaxy $\left(\alpha / \alpha_{\mathrm{Gal}}=40-100\right.$, Israel 1997; Madden et al. 1997). However, the conversion factor has been directly measured on smaller scales for the regions that we are studying by Wilson (1995), and so we adopt her values of $\alpha / \alpha_{\mathrm{Gal}}=2.7 \pm 0.5$ for IC 10 and $2.2 \pm 0.8$ for NGC 6822 and note that this gives the lower limit on the mass of the molecular gas. We use $34.6 \mathrm{Jy} \mathrm{K}^{-1}\left(\eta_{\mathrm{ap}}=0.45\right)$ and $29.4 \mathrm{Jy} \mathrm{K}^{-1}\left(\eta_{\mathrm{ap}}=0.53\right)$ to convert the JCMT data from kelvins $\left(T_{\mathrm{A}}^{*}\right)$ to janskys in IC 10 and NGC 6822, 
respectively (Kraus 1986). We assume a coupling efficiency $\left(\eta_{\mathrm{c}}\right)$ of 0.7 to correct our observed fluxes to true fluxes. The virial masses from Becker (1990) were corrected to the new IC 10 distance of $0.82 \mathrm{Mpc}$. The $\mathrm{CO}-$ to- $\mathrm{H}_{2}$ conversion factor is only accurate to within $\sim 30 \%$, while our fluxes are typically accurate to about $10 \%$. We therefore adopt a total uncertainty of $40 \%$. The results are shown in Table 2.

It is interesting that the masses calculated here for the IC 10 regions are larger than the virial masses $\left(M_{\mathrm{vir}}\right)$ found by Becker (1990). Our beam size of 22" covers a diameter of 88 $\mathrm{pc}$ at the distance of IC 10. Although this beam is smaller than the dimensions of the clouds in IC 10 as measured by Becker using a comparable (21") beam size (e.g., IC 10:MC $1 / 2$ measured $107 \mathrm{pc} \times 98 \mathrm{pc}$ ), the IRAM dimensions are not much larger than the beam and were not deconvolved from the beam. Thus, our single-position measurements probably detect most of the emission in each region. Given the large uncertainties, the molecular mass of all the clouds in IC 10 lie within $2 \sigma$ of the virial masses calculated by Becker (1990). If the clouds were not gravitationally bound, the virial masses would be larger than the molecular masses. We thus conclude that these quite large structures are gravitationally bound.

The mass of IC 10: MC 1/2 $\left(\sim 5 \times 10^{6} M_{\odot}\right)$ indicates that it may be either a large giant molecular cloud (GMC; $M=10^{5}-10^{6} M_{\odot}$; Sanders, Scoville, \& Solomon 1985) or a small giant molecular association (GMA; $M=10^{7}-10^{8}$ $M_{\odot}$; Rand \& Kulkarni 1990). For star formation to occur, we would naturally expect that the molecular clouds in which the stars form must be gravitationally bound. All of the GMCs observed in IC 10 appear to be gravitationally bound (Wilson \& Reid 1991; Wilson 1995). The larger region IC 10:MC 1/2 also appears to be gravitationally bound. Small GMAs seen in M33 all appear unbound by factors of about 5, except in giant $\mathrm{H}$ iI regions (Wilson \& Scoville 1989, 1992). In M51, the star formation efficiency is enhanced in the spiral arms where GMAs are seen (Rand \& Kulkarni 1990). Individual GMCs inside the potential wells of the bound GMAs may be colliding at a much higher rate than isolated GMCs, which could increase the star formation rate and the star formation efficiency. Thus the presence of large bound molecular structures in IC 10 may provide a possible explanation for the high star formation rate in IC 10.

For NGC 6822: MC 2, the molecular mass is a factor of 3.4 larger than the virial mass calculated by Wilson (1994) using the Owens Valley Millimeter-Wave Interferometer. This large mass measurement may be caused by the larger $22^{\prime \prime}$ beam overlapping portions of the nearby cloud (NGC 6822:MC 1), which is approximately $17^{\prime \prime}$ away. Also, the virial masses for NGC 6822 are taken from interferometer data, which are insensitive to diffuse structures that can be detected by single dishes such as the JCMT. Finally, in determining the molecular mass for NGC 6822:MC 2, we assumed a ${ }^{12} \mathrm{CO} J=2-1 / J=1-0$ value of 0.7 . Given the rather high ${ }^{12} \mathrm{CO} J=3-2 / J=2-1$ ratio, we might expect this cloud to have an unusually high ${ }^{12} \mathrm{CO} J=2-1 / J=1-0$ ratio as well. Increasing the ${ }^{12} \mathrm{CO} J=2-1 / J=1-0$ line ratio to a value of $\sim 1.0$ would give molecular and virial mass estimates that agree within the uncertainties.

The CO-to- $\mathrm{H}_{2}$ conversion factor relative to the Galactic value is determined by assuming the clouds are virialized and setting the molecular mass $M_{\mathrm{mol}}$ from equation (1) equal to the virial mass $M_{\text {vir }}$. This method gives the relation $M_{\text {vir }} / M_{\text {mol }}=\alpha / \alpha_{\text {Gal }}$ (e.g., Wilson 1995). Equation (1) assumes that the gas is optically thick and that emission comes from the surface. However, MC 2 in NGC 6822 appears to be optically thin. For an optically thin cloud, the emission from the entire volume of the cloud can escape, and we can get a much higher flux. This effect would result in an overestimate of the molecular mass found using equation (1), which in turn would result in an underestimate of $\alpha / \alpha_{\mathrm{Gal}}$. Even if only part of the cloud were optically thin, we would still be overestimating molecular masses. Because the clouds in NGC 6822 were not fully resolved, the derived virial masses were only upper limits, and hence the value of $\alpha / \alpha_{\mathrm{Gal}}$ derived by Wilson (1994) is also an upper limit. Since NGC 6822:MC 2 is optically thin, we now see that $M_{\text {mol }}$ was also overestimated, which would work to balance the previous uncertainty and make the value of $\alpha / \alpha_{\mathrm{Gal}}$ found by Wilson (1994) more reliable. However, since we do not know the extent of each mass overestimate, we cannot make precise corrections to the previously published values of $\alpha$.

Given that the ${ }^{12} \mathrm{CO}$ emission from the cloud NGC 6822:MC 2 is optically thin, we have a rare opportunity to calculate the mass of the cloud directly. Using the upper limit on column density determined from the LVG analysis, we can estimate the mass by adopting a number density ratio of $n(\mathrm{CO}) / n\left(\mathrm{H}_{2}\right)=10^{-4}$ (Genzel 1992) and multiplying by the area of the cloud $\pi a b$, where $a$ and $b$ are the major and minor axes of the elliptical cloud. It is probably most appropriate to use the dimensions of the cloud obtained in the interferometry maps $(36 \times<13$ pc, Wilson 1994) instead of the JCMT beam size. The LVG model indicates that the CO column density can be at most $5.3 \times 10^{17}$ $\mathrm{cm}^{-2}$ (at temperatures of $100 \mathrm{~K}$ ) to produce the observed line ratio, which leads to a total mass of $\mathrm{H}_{2}$ of less than $1.2 \times 10^{5} M_{\odot}$. This mass is in good agreement with the molecular mass derived using equation (1) and agrees with the virial mass to within a factor of 2 . As discussed in $\S 3.3$,

TABLE 2

Masses of Molecular Clouds in IC 10 AND NGC 6822

\begin{tabular}{|c|c|c|c|}
\hline Cloud & $\begin{array}{c}S_{\mathrm{CO}(J=2-1)} \\
\left(\mathrm{K} \mathrm{km} \mathrm{s}^{-1}\right)\left(T_{\mathrm{A}}^{*}\right)\end{array}$ & $\begin{array}{c}\text { Molecular } \text { Mass }^{\mathrm{a}} \\
\left(M_{\odot}\right)\end{array}$ & $\begin{array}{l}\text { Virial Mass }{ }^{\mathrm{b}} \\
\qquad\left(M_{\odot}\right)\end{array}$ \\
\hline IC $10: \mathrm{MC} 1 / 2$ & 9.16 & $5.2 \times 10^{6}$ & $2.5 \times 10^{6}$ \\
\hline IC $10: R 2 \ldots \ldots$ & 2.67 & $0.83 \times 10^{6}$ & $0.35 \times 10^{6}$ \\
\hline IC $10:$ MC $4 \ldots$ & 2.37 & $1.3 \times 10^{6}$ & $0.54 \times 10^{6}$ \\
\hline IC $10:$ MC $6 / 7 / 8 \ldots \ldots \ldots$ & 2.35 & $1.7 \times 10^{6}$ & $0.66 \times 10^{6}$ \\
\hline NGC 6822:MC $2^{\mathrm{c}} \ldots \ldots$ & 1.66 & $0.21 \times 10^{6}$ & $<6.3 \times 10^{4}$ \\
\hline
\end{tabular}

${ }^{a}$ A CO-to- $\mathrm{H}_{2}$ conversion factor $\left(\alpha / \alpha_{\text {Gal }}\right)$ of 2.7 and 2.2 has been assumed for IC 10 and NGC 6822, respectively.

b Virial masses taken from Becker 1990, except NGC 6822:MC 2 (Wilson 1994).

${ }^{c} \mathrm{~A}{ }^{12} \mathrm{CO} J=2-1 / J=1-0$ ratio of 0.7 has been assumed. 
we expect the mass derived from single-dish flux to be overestimated. Since the virial mass is comparable to the mass derived from the column density, it is likely that the molecular cloud MC 2 in NGC 6822 is gravitationally bound.

\section{IMPLICATIONS FOR THE CO-TO-H ${ }_{2}$ CONVERSION FACTOR}

It has been proposed that the $\mathrm{CO}-$ to- $\mathrm{H}_{2}$ conversion factor $(\alpha)$ is not only dependent on density and brightness temperature $\left(\propto \rho^{1 / 2} / T_{b}\right)$, but also on the metallicity (Dickman, Snell, \& Schloerb 1986; Maloney \& Black 1988; Sakamoto 1996). It is possible that the lower metallicity in irregular galaxies may change the typical temperatures and densities of the molecular clouds they contain. Although this effect may confuse any observed relation between $\alpha$ and metallicity, in essence it will simply introduce interrelationships between $\rho, T_{b}$, and metallicity. Overall, the expression for $\alpha$ will still be a function of metallicity.

The line ratios for the clouds in IC 10 agree with those found in M33 by Wilson et al. (1997) within the measurement uncertainties (see Table 1). If we assume that the line widths are the same for both transitions, then the line ratios are essentially ratios of radiation temperature $T_{R}$ (e.g., Walker 1991). The radiation temperature is related to the cloud excitation temperature and density by

$$
T_{R}=\left[J_{v}\left(T_{\mathrm{ex}}\right)-J_{v}\left(T_{\mathrm{bg}}\right)\right]\left(1-e^{-\tau}\right),
$$

where $J_{v}(T)$ is the Planck function, $T_{\mathrm{bg}}$ is the background temperature, and $T_{\mathrm{ex}}$ is the excitation temperature of the cloud. The optical depth of the cloud is given by

$$
\tau=\int_{0}^{D} K \rho d r
$$

where $D$ is the depth of the cloud, $\rho$ is the density profile of the cloud, and $K$ is the absorption coefficient. For the optically thin case where $\tau \ll 1$, the ${ }^{12} \mathrm{CO} J=3-2 / J=2-1$ line ratio becomes

$$
\frac{T_{R}^{3-2}}{T_{R}^{2-1}}=\frac{f^{3-2}\left[J_{v}\left(T_{\mathrm{ex}}^{3-2}\right)-J_{v}\left(T_{\mathrm{bg}}\right)\right] \tau^{3-2}}{f^{2-1}\left[J_{v}\left(T_{\mathrm{ex}}^{2-1}\right)-J_{v}\left(T_{\mathrm{bg}}\right)\right] \tau^{2-1}},
$$

where $f$ is the beam filling factor of the emission, and the superscript 3-2 indicates those values pertaining to the $J=3-2$ transition. For the optically thick case, $\tau \gg 1$, the line ratio has the form

$$
\frac{T_{R}^{3-2}}{T_{R}^{2-1}}=\frac{f^{3-2}\left[J_{v}\left(T_{\mathrm{ex}}^{3-2}\right)-J_{v}\left(T_{\mathrm{bg}}\right)\right]}{f^{2-1}\left[J_{v}\left(T_{\mathrm{ex}}^{2-1}\right)-J_{v}\left(T_{\mathrm{bg}}\right)\right]} .
$$

Both these line ratios are a complicated function of the excitation temperature. Thus, to produce similar line ratios in clouds with different physical conditions would require a pathological interdependence of density and temperature to produce the same excitation temperature for the low- $J$ CO transitions. We feel that the similarity in the line ratios in the clouds in IC 10 and M33 indicates similar physical conditions (namely similar $\rho$ and $T$ ), and thus the difference in the measured value of $\alpha$ (Wilson 1995) can be attributed to the difference in metallicity of the spiral galaxy M33 and the metal-poor dwarf irregulars IC 10 and NGC 6822.

\section{CONCLUSIONS}

We have observed the ${ }^{12} \mathrm{CO} J=2-1$ and $J=3-2$ lines at a few locations in the dwarf irregular galaxies IC 10 and NGC 6822. In addition, we have observed the ${ }^{13} \mathrm{CO}$
$J=2-1$ and $J=3-2$ lines for molecular clouds in IC 10 . The clouds are located in a variety of star formation environments. In general, the clouds in IC 10 show line ratios similar to those found in M33 by Wilson et al. (1997). We find that the ${ }^{12} \mathrm{CO} J=3-2 / J=2-1$ ratio increases with increasing proximity to $\mathrm{H}$ II regions, as previously found by Wilson et al. (1997) in M33. This result may indicate a general relation that holds true for all galaxies (and galaxy types) and is likely the result of a combination of extreme temperature and density conditions near star-forming regions. In this low-metallicity (and in NGC 6822, low-CO column density) environment, this effect is more conspicuous than it is in the higher metallicity spiral galaxy M33.

Combining the JCMT line ratios in IC 10:MC $1 / 2$ with large velocity gradient models indicates that this region has a kinetic temperature of $100 \mathrm{~K}$, densities of $10^{4}-10^{5} \mathrm{~cm}^{-3}$ and a column density of $6 \times 10^{18} \mathrm{~cm}^{-2}$. The $\left[{ }^{12} \mathrm{CO}\right] /$ $\left[{ }^{13} \mathrm{CO}\right]$ abundance is between 50 and 70 . The inclusion of the IRAM data from Becker (1990) suggests that there are two distinct emitting regions, a high-density region where the upper $J$ transitions dominate, and a lower density region where the lower $J$ transitions dominate. For IC $10: \mathrm{MC} 6 / 7 / 8$, if we assume a temperature of $20 \mathrm{~K}$ and $\left[{ }^{12} \mathrm{CO}\right] /\left[{ }^{13} \mathrm{CO}\right]=50$, we obtain densities of $10^{1.6}-10^{4}$ $\mathrm{cm}^{-3}$ and column densities of $2 \times 10^{17}-2 \times 10^{19} \mathrm{~cm}^{-2}$.

We find an unusually high ${ }^{12} \mathrm{CO} J=3-2 / J=2-1$ line ratio in NGC 6822 that we attribute to optically thin CO emission resulting from the combination of a low molecular gas content and a low-metallicity environment. An LVG analysis of the ${ }^{12} \mathrm{CO} J=3-2 / J=2-1$ line ratio in NGC 6822: MC 2 indicates the presence of hot $(T \geq 100 \mathrm{~K})$ optically thin gas. The density of this cloud is likely greater than $10^{4.2} \mathrm{~cm}^{-3}$, and its column density is less than $5 \times 10^{17}$ $\mathrm{cm}^{-2}$.

The optically thin CO emission in NGC 6822 allows a rare opportunity to calculate the mass of molecular gas directly. Mass calculations from two separate techniques determine the molecular mass to be $1.2-2.1 \times 10^{5} M_{\odot}$. This agrees with the virial mass to within a factor of $\sim 3$. Flux measurements yield masses for the cloud complexes in IC 10 that indicate that these regions are gravitationally bound. The masses of the clouds range from $0.83-5.2 \times 10^{6}$ $M_{\odot}$. These regions may represent either large giant molecular clouds or small giant molecular associations. The presence of these large gravitationally bound structures may help explain the high star formation rate in IC 10.

The similarity in the line ratios between IC 10 and M33 is most easily understood if the physical conditions in these clouds are also similar, in which case any variation in the CO-to- $\mathrm{H}_{2}$ conversion factor between these two galaxies can be attributed to differences in their metallicity. The increase in the ${ }^{12} \mathrm{CO} J=3-2 / J=2-1$ ratio near star forming regions may be a result of either higher temperatures, higher densities, or some combination of the two. Upcoming SCUBA observations will provide temperature measurements that will enable us to determine better the physical processes at work.

The JCMT is operated by the Royal Observatories on behalf of the Particle Physics and Astronomy Research Council of the United Kingdom, the Netherlands Organization for Scientific Research, and the National Research Council of Canada. This research has been supported by a research grant to C. D. W. from NSERC (Canada). 


\section{REFERENCES}

Arnault, P., Casoli, F., Combes, F., \& Kunth, D. 1988, A\&A, 205, 41

Becker, R. 1990, Ph.D. thesis, Univ. Bonn

Combes, F. 1986, in Star-Forming Dwarf Galaxies and Related Objects, ed. D. Kunth, T. X. Thaun, \& J. Tran Thanh Van (Singapore: Kim Hup Lee), 307

de Vaucouleurs, G., \& Ables, H. 1965, PASP, 77, 272

Dickman, R. L., Snell, R. L., \& Schloerb, F. P. 1986, ApJ, 309, 326

Elmegreen, B. G. 1989, ApJ, 338, 178

Genzel, R. 1992, in The Galactic Interstellar Medium, ed. D. Pfenniger and P. Bartholdi (Berlin: Springer), 275

Herbig, G. H., \& Terndrup, D. M. 1986, ApJ, 307, 609

Hodge, P. W., Kennicutt, R. C., \& Lee, M. G. 1988, PASP, 100, 917

Hodge, P. W., \& Lee, M. G. 1990, PASP, 102, 26

Hodge, P. W., Lee, M. G., \& Kennicutt, R. C. 1989, PASP, 101, 32

Hubble, E. P. 1925 , ApJ, 62, 409

Israel, F. P. 1997, A\&A, 317, 65

Israel, F. P., et al. 1993, A\&A, 276, 25

Kennicutt, R. C. 1988, ApJ, 334, 144

Kraus, J. D. 1986, Radio Astronomy (2d ed.; Ohio: Quasar-Cygnus)

Lequeux, J., Peimbert, M., Rayo, J. F., Serrano, A., \& Torres-Peimbert, S. 1979, A\&A, 80, 155

Madden, S. C., Poglitsch, A., Geis, N., Stacey, G. J., \& Townes, C. H. 1997, ApJ, 483, 200

Maloney, P., \& Black, J. H. 1988, ApJ, 325, 389

Massey, P., \& Armandroff, T. E. 1995, AJ, 109, 2470

McGonegal, R., McLaren, R. A., Welch, D. L., Madore, B. F., \& McAlary, C. W. 1983, ApJ, 273, 539

McLaughlin, D. E., \& Pudritz, R. E. 1996, ApJ, 469, 194

Mouschovias, T., \& Spitzer, L. 1976, ApJ, 210, 326
Ohta, K., Sasaki, M., Yamada, T., \& Saitō, M. 1992, PASJ, 44, 585

Rand, R. J., \& Kulkarni, S. R. 1990, ApJ, 349, L43

Sakamoto, S. 1996, ApJ, 462, 215

Sandage, A., \& Tammann, G. A. 1974, ApJ, 194, 559

Sanders, D. B., Scoville, N. Z., \& Solomon, P. M. 1985, ApJS, 289, 373

Schmidt, K.-H., \& Boller, T. 1993, Astron. Nachr., 314, 5, 361

Scoville, N. Z., \& Sanders, D. B. 1987, in Interstellar Processes, ed. D. J. Hollenbach \& H. A. Thronson (Dordrecht: Reidel), 21

Scoville, N. Z., \& Solomon, P. M. 1974, ApJ, 187, L63

Shostak, G. S., \& Skillman, E. D. 1989, A\&A, 214, 33

Solomon, P. M., Rivolo, A. R., Barrett, J., \& Yahil, A. 1987, ApJ, 319, 730

Strong, A. W., et al. 1988, A\&A, 207,1

Stutzki, J., \& Güsten, R. 1990, ApJ, 356, 513

Tacconi, L. J., \& Young, J. S. 1987, ApJ, 332, 681

Thornley, M. D., \& Wilson, C. D. 1994, ApJ, 421, 458

Tilanus, R. P. J., Tacconi, L. J., Sutton, E. C., Zhou, S., Sanders, D. B., Wynn-Williams, C. G., Lo, K. Y., \& Stephens, S. A. 1991, ApJ, 376, 500

Vilchez, J. M., Pagel, B. E. J., Diaz, A. I., Terlevich, E., \& Edmunds, M. G. 1988, MNRAS, 235, 633

Walker, C. E. 1991, Ph.D. thesis, Univ. Arizona

Wilson, C. D. 1994, ApJ, 434, L11

. 1995, ApJ, 448, L97

Wilson, C. D., \& Reid, I. N. 1991, ApJ, 366, L11

Wilson, C. D., \& Scoville, N. Z. 1989, ApJ, 347, 743 . 1990, ApJ, 363, 435 1992, ApJ, 385, 512

Wilson, C. D., Walker, C. E., \& Thornley, M. D. 1997, ApJ, 483, 210

Wilson, C. D., Welch, D. L., Reid, I. N., Saha, A., \& Hoessel, J. 1996, AJ, 111,1106 